\title{
Editorial
}

\section{Improving Neurodevelopmental Outcomes After Neonatal Herpes Simplex Virus Infection}

\section{William C. Golden, MD}

Journal of Perinatology (2005) 25, 154-155. doi:10.1038/sj.jp.7211236

Approximately 1500 newborns acquire herpes simplex virus (HSV) infections annually. The severity of neonatal HSV disease is clearly reflected in one of the first case reports of the entity - a premature infant who died with significant hepatic and adrenal necrosis. ${ }^{1}$ Over the past 20 years, the evolution of antiviral therapy (vidarabine to high-dose acyclovir) and the use of polymerase chain reaction (PCR) to detect HSV DNA have greatly improved our ability to diagnose and manage neonatal herpes infections. Despite these advances, recent data indicate that the interval between detection of HSV symptoms and initiation of antiviral therapy in affected infants has not changed since the introduction of acyclovir. ${ }^{2}$ Furthermore, neonates with HSV encephalitis and disseminated $\mathrm{HSV}^{2}$ as well as those with recurrent infections ${ }^{3}$ are at higher risk of abnormal neurological development. Thus, neonatologists and primary care pediatricians are charged with the task of reducing the severity, mortality, and long-term morbidities of infants with HSV.

In this issue of the Journal, Tiffany et al. ${ }^{4}$ describe favorable neurodevelopmental outcomes in a small cohort of infants receiving long-term, high-dose acyclovir after initial treatment for either disseminated or CNS HSV infection. After completing 2 years of oral suppressive therapy, 11 of 16 infants had no evidence of developmental delay at 2 years of age. Additionally, when stratified based on risk factors for poor neurodevelopmental outcome (prematurity, HSV two infection, evidence of CNS involvement), $50 \%$ or more of infants in each category showed normal or accelerated motor and/or cognitive development.

Recurrence of cutaneous HSV after initial neonatal infection is associated with the development of neurologic deficits, ${ }^{5}$ and HSV DNA has been documented in the CSF of infants with mucocutaneous, encephalitic, and disseminated herpes. ${ }^{6}$ In one

The Johns Hopkins University School of Medicine (W.C.G.), Baltimore, MD, USA

Address correspondence and reprint requests to William Christopher Golden, MD, The Johns Hopkins University, School of Medicine, 600 North Wolfe Street, Nelson 2-133, Baltimore, MD 21287, USA multicenter trial, suppressive acyclovir reduced recurrence of dermal lesions in infants with prior mucocutaneous HSV ("SEM disease"). ${ }^{7}$ The current study extends the rationale for long-term suppression by quantifying improved neurological outcome in patients with the most severe forms of neonatal HSV.

Should we institute suppressive acyclovir therapy routinely after the standard 14- or 21-day treatment course for neonatal HSV? Such a significant change in management strategy at this time is premature. Tiffany and colleagues astutely highlight the limitations of their study and indicate the need for further evaluation of this therapeutic intervention. Indeed, a randomized controlled trial (long-term acyclovir versus placebo) would allow for more precise delineation of changes in neurodevelopmental outcomes after suppressive therapy. In addition, the benefits of long-term acyclovir may be transient; infants on antiviral suppression should have additional, extensive follow-up beyond the window of therapy to assess for recurrence of HSV and delays in achievement of developmental milestones.

Further studies should also focus on the interplay between longterm acyclovir, maturation of the neonatal immune system, and neurodevelopmental outcome. In the current study, five of the 16 infants had developmental delays despite appropriate serum acyclovir concentrations. Did these infants have lower levels of maternally acquired antibodies than their normal counterparts? Did the infants fail to mount an appropriate antibody response in the face of suppressive therapy, thus rendering them susceptible to recurrent infections? Infants treated with standard, high-dose intravenous acyclovir for active neonatal HSV had normal antibody titers during and after therapy; ${ }^{8}$ however, the humoral and cellular response to extended antiviral therapy is not known. In addition to monitoring for adverse drug reactions, any clinical trial of longterm acyclovir use should include quantification of the immune response in treated neonates, documentation of subclinical CNS disease (via HSV PCR, and/or MRI), and the potential for development of antiviral resistance. Such information would prove useful in understanding the host immune response, the appropriate length of therapy to ensure favorable long-term outcomes, and characteristics of infants who may not respond to suppressive therapy.

In addition to studying the use of long-term acyclovir, current, established strategies must also be employed to decrease the incidence of neonatal HSV infection. Pediatricians must always include congenital herpes in the differential diagnosis of neonatal sepsis and consider screening tests for HSV (i.e., viral cultures, 
aminotransferase levels, coagulation studies) and empiric acyclovir therapy in critically ill infants. Obstetricians should employ maternal acyclovir prophylaxis in women with a history of HSV to reduce recurrent lesions and/or asymptomatic shedding at delivery. With the recent commercial availability of type-specific HSV antibody assays, obstetricians also should screen pregnant women to identify patients with new-onset primary infections (who pose the greatest vertical transmission risk ${ }^{9}$ ) and those women at risk for acquiring HSV during pregnancy. While mitigating the sequelae of infection remains an important goal, prevention of neonatal HSV disease remains the only means of avoiding subsequent neurodevelopmental morbidities and death.

\section{References}

1. Haas G. Hepato-adrenal necrosis with intranuclear inclusion bodies: report of a case. Am J Pathol 1935;11:127-47.

2. Kimberlin DW, Lin CY, Jacobs RF, et al. The National Institute of Allergy and Infectious Diseases Collaborative Antiviral Study Group. Natural history of neonatal herpes simplex virus infections in the acyclovir era. Pediatrics 2001;108(2):223-9.

3. Kimura H, Futamura M, Ito $\mathrm{Y}$, et al. Relapse of neonatal herpes simplex virus infection. Acrh Dis Child Fetal Neonatal Ed 2003;88(6):F483-6.
4. Tiffany KF, Benjamin Jr DK, Palisanthiran P, O'Donnell K, Gutman LT. Improved neurodevelopmental outcomes following long-term high-dose acyclovir therapy in infants with disseminated /encephalitic herpes simplex disease. J Perinatol 2005;25(3):156-61.

5. Whitley R, Arvin A, Prober C, et al. The National Institutes of Allergy and Infectious Diseases Collaborative Antiviral Study Group. Predictors of morbidity and mortality in neonates with herpes simplex virus infections. $\mathrm{N}$ Engl J Med 1991;324(7):450-4.

6. Kimberlin DW, Lakeman FD, Arvin AM, et al. The National Institutes of Allergy and Infectious Diseases Collaborative Antiviral Study Group. Application of the polymerase chain reaction to the diagnosis and management of neonatal herpes simplex virus disease. J Infect Dis 1996;174(6):1162-7.

7. Kimberlin D, Powell D, Gruber W, et al. The National Institutes of Allergy and Infectious Diseases Collaborative Antiviral Study Group. Administration of oral acyclovir suppressive therapy after neonatal herpes simplex virus disease limited to the skin, eyes and mouth: results of a phase I/II trial. Pediatr Infect Dis J 1996;15(3):247-54.

8. Kimberlin DW, Lin CY, Jacobs RF, et al. National Institute of Allergy and Infectious Diseases Collaborative Antiviral Study Group. Safety and efficacy of high-dose intravenous acyclovir in the management of neonatal herpes simplex virus infections. Pediatrics 2001;108(2):230-8.

9. Kimberlin DW. Neonatal herpes simplex infection. Clin Microbiol Rev 2004;17(1):1-3. 